were evaluated against the clinical diagnosis of SLE as judged by the expert panel on the sensitivity and specificity, which was calculated by $2 \times 2$ contingency tables ("condition positive" = clinical diagnosis of SLE; "test positive" = criteria positive for SLE) with standard formulas (sensitivity = true positive/[true positive + false negative]; specificity = true negative / [true negative + false positive]). Receiver operating characteristic (ROC) curve was used to study the optimal cutoff points from the EULAR/ACR criteria for the highest summation of specificity and sensitivity.

Results: 3967 patients were screened; 1542 patients who were positive for ANA ( $\geq 1: 80)$ were included ( $88.3 \%$ women). The mean age of these patients at first rheumatology clinic attendance was $45.6 \pm 15.0$ years and the duration of follow-up was $7.5 \pm 7.0$ years. A total of 567 patients were judged to have SLE by the expert panel (discrepancy of clinical diagnosis in 135 patients resolved with voting). The sensitivity and specificity of the three SLE classification criteria in our patients are listed in Table 1. ROC analysis showed that the best cut-off for a clinical diagnosis of SLE using the EULAR/ACR criteria was 10 points (area under the curve [AUC] 0.977 ; sensitivity $89.2 \%$ and specificity $89.6 \%$ ). Similar figures were obtained for subgroups of patients stratified by gender and different age ranges.

Conclusion: In our cohort of Hong Kong Chinese patients, the 2019 EULAR/ ACR criteria is more sensitive but less specific when compared with 1997 ACR criteria for classifying SLE. On the other hand, the EULAR/ACR criteria is less sensitive but more specific than the 2012 SLICC criteria. The specificity of the EULAR/ACR criteria for SLE is higher in male than female patients. In our patients older than 50 years, the EULAR/ACR criteria is less sensitive but more specific for a classification of SLE. Overall, the performance of the EULAR/ACR criteria for a diagnosis of SLE in our study is similar to that reported in recent Asian studies although the sensitivity is lower, which may be related to the inclusion of ANA+ patients only. REFERENCES:

\begin{tabular}{lll}
\hline Classification criteria & Sensitivity & Specificity \\
\hline 1997 ACR & $85.9 \%$ & $94.4 \%$ \\
2012 SLICC & $97.5 \%$ & $86.4 \%$ \\
2019 EULAR/ACR with 10 points as cut-off & $89.2 \%$ & $89.6 \%$ \\
2019 EULAR/ACR with 9 points as cut-off & $93.6 \%$ & $68.7 \%$ \\
2019 EULAR/ACR with 11 points as cut-off & $86.9 \%$ & $92.4 \%$ \\
2019 EULAR/ACR with 10 points as cut-off (men) & $88.9 \%$ & $94.5 \%$ \\
2019 EULAR/ACR with 10 points as cut-off (women) & $89.2 \%$ & $88.8 \%$ \\
2019 EULAR/ACR with 10 points as cut-off (age >50 years) & $78.7 \%$ & $94.1 \%$ \\
2019 EULAR/ACR with 10 points as cut-off (age $\leq 50$ years) & $91.7 \%$ & $84.1 \%$ \\
\hline
\end{tabular}

Disclosure of Interests: None declared

DOI: 10.1136/annrheumdis-2021-eular.3394

\section{POS0772 \\ PREVALENCE AND RISK FACTORS FOR HERPES ZOSTER REACTIVATION IN 1542 PATIENTS WITH RHEUMATIC DISEASES}

C. C. Mok ${ }^{1}$, L. Y. Ho ${ }^{1}$, S. M. Tse ${ }^{1}$, K. L. Chan ${ }^{1}$, C. H. To ${ }^{2} .{ }^{1}$ Tuen Mun Hospital, Medicine, Hong Kong, Hong Kong (SAR); ${ }^{2}$ Pok Oi Hospital, Medicine, Hong Kong, Hong Kong (SAR)

Objectives: To study the prevalence and risk factors of herpes zoster $(\mathrm{HZ})$ infection in patients with rheumatic diseases.

Methods: Medical records of patients with rheumatic diseases who attended our out-patient rheumatology clinics between 2019 March and 2019 August were retrospectively reviewed. Patients who were using biological or targeted DMARDs were excluded. Episodes of $\mathrm{HZ}$ infection since disease diagnosis were identified and the prevalence over time was calculated. Laboratory parameters (total white cell count, neutrophil-to-lymphocyte ratio, serum albumin, globulin \& creatinine), history of diabetes mellitus and the highest doses of immunosuppressive medications within 6 months of the first episode of $\mathrm{HZ}$ infection were compared with those within 6 months of last follow-up in patients who did not have $\mathrm{HZ}$ infection. Cox regression analysis was performed to identify factors associated with the first $\mathrm{HZ}$ infection in all patients.

Results: 1542 patients were studied ( $88 \%$ women, age $46.4 \pm 15.0$ years). The underlying diseases were systemic lupus erythematosus (SLE) $(38 \%)$, rheumatoid arthritis (26\%) and other rheumatic diseases (36\%). After a total follow-up of 11,515 patient-years since diagnosis $(7.5 \pm 7.0$ years), $122(7.9 \%)$ patients developed 146 episodes of $\mathrm{HZ}$ infection, giving an overall prevalence of 1.27/100-patient years. The prevalence rates of $\mathrm{HZ}$ in SLE, RA and non-SLE/RA patients were $1.70,0.64$ and 0.76 per 100 patient-years, respectively. Patients who experienced $\mathrm{HZ}$ reactivation were younger $(41.6 \pm 14.7$ vs $46.8 \pm 15.0$ years; $p<0.001)$, more likely to have SLE ( $74 \%$ vs $35 \%$; $p<0.001)$ and diabetes mellitus $(17 \%$ vs $7.3 \%$; $\mathrm{p}=0.01$ ), and had a significantly lower albumin (38.6 \pm 5.6 vs $41.3 \pm 3.5 ; \mathrm{p}<0.001)$ and higher neutrophil-to-lymphocyte ratio $(4.9 \pm 6.2$ vs $2.8 \pm 2.6 ; p<0.001)$. More patients with $\mathrm{HZ}$ reactivation were treated with prednisolone (54\% vs $22 \%$; $\mathrm{p}<0.001)$, azathioprine ( $20 \%$ vs $8 \%$; $\mathrm{p}<0.001)$, mycophenolate mofetil [MMF] ( $21 \%$ vs $12 \% ; p=0.006)$, cyclophosphamide [CYC] $(4.9 \%$ vs $0.1 \% ; p<0.001)$ and hydroxychloroquine ( $48 \%$ vs $34 \% ; p=0.002$ ) in the preceding 6 months compared with those who did not have $\mathrm{HZ}$ infection. Among those using immunosuppressive drugs, the doses of MMF $(1.42 \pm 0.64$ vs $1.02 \pm 0.31 \mathrm{~g} ; \mathrm{p}=0.005)$ and prednisolone $(15.6 \pm 15.9$ vs $5.5 \pm 4.5 \mathrm{mg} ; \mathrm{p}<0.001)$ were significantly higher in those with $\mathrm{HZ}$ infection. The cumulative risk of having $\mathrm{HZ}$ reactivation in SLE patients at 24 and 48 months since diagnosis was $5.9 \%$ and $8.6 \%$, respectively, which was significantly higher than that in non-SLE patients $(1.9 \%$ and $2.5 \%$, respectively; $\mathrm{p}<0.001$ by log rank test). Cox regression analysis revealed that having a diagnosis of SLE (HR 1.97 [1.17-3.31]), albumin level (HR 0.93 [0.90-0.97] per $\mathrm{g} / \mathrm{L} ; \mathrm{p}=0.001$ ), serum creatinine (HR 0.995 [0.990-1.00] per umol/L), higher neutrophil/lymphocyte ratio (HR 1.08 [1.05-1.11]) and the use of CYC (HR 6.69 [2.5617.5]) and prednisolone (HR 1.61 [1.02-2.45]) in the preceding 6 months were independently associated with the development of $\mathrm{HZ}$ infection.

Conclusion: Reactivation of $\mathrm{HZ}$ is fairly common in patients with rheumatic diseases. Underlying SLE, prednisolone/cyclophosphamide therapy and the neutrophil/lymphocyte ratio, but not age, sex or other laboratory parameters, are the major risk factors for $\mathrm{HZ}$ reactivation.

Disclosure of Interests: None declared

DOI: 10.1136/annrheumdis-2021-eular.3398

\section{POS0773 \\ CLINICAL PRESENTATION AND OUTCOMES OF THE ANTIPHOSPHOLIPID SYNDROME (APS) IN SOUTHERN CHINESE PATIENTS: THE HONG KONG APS REGISTRY}

C. C. Mok ${ }^{1}$, C. Lee ${ }^{2}$, M. C. Leung ${ }^{3}$, Y. K. Tang ${ }^{4}$, C. Ho ${ }^{5}$, S. Woo ${ }^{6}$, M. L. Lee ${ }^{7}$, M. C. Wan ${ }^{6}$, M. Y. Lee ${ }^{8}$ on behalf of Hong Kong APS registry working group. ${ }^{1}$ Tuen Mun Hospital, Medicine, Hong Kong, Hong Kong (SAR); ${ }^{2}$ Pok Oi Hospital, Medicine, Hong Kong, Hong Kong (SAR); ${ }^{3}$ United Christian Hospital, Medicine, Hong Kong, Hong Kong (SAR); ${ }^{4}$ Kwong Wah Hospital, Medicine, Hong Kong, Hong Kong (SAR); ${ }^{5}$ Queen Mary Hospital, Medicine, Hong Kong, Hong Kong (SAR); ${ }^{6}$ Rutonjee Hospital, Medicine, Hong Kong, Hong Kong (SAR); ${ }^{7}$ Yan Chai Hospital, Medicine, Hong Kong, Hong Kong (SAR); ${ }^{8}$ Tai Po Hospital, Medicine, Hong Kong, Hong Kong (SAR)

Objectives: To report the clinical presentation and outcomes of the APS in Hong Kong Chinese patients.

Methods: The HKAPS registry was established in early 2020 by the Hong Kong Society of Rheumatology to study the outcomes of Chinese patients with APS treated in public hospitals in Hong Kong. Patients aged $\geq 18$ years were identified by the Hospital Authority Clinical Data retrieval system using the ICD-10 diagnostic code of APS. The medical history and APS diagnosis was verified by sub-investigators in different hospitals using the 2006 modified consensus criteria for the APS. Eligible patients were classified into definite APS or probable APS, which was further categorized into primary (without underlying rheumatic diseases) and secondary types. The presentation, treatment and outcomes of these patients were summarized.

Results: 232 APS patients (76.3\% women) were identified. All were ethnic Chinese. A total of 160 patients fulfilled the 2006 criteria for APS (definite APS) while 72 patients had probable APS (anti-phospholipid [aPL] antibodies positive once or with non-criteria manifestations). In those with definite APS, the mean age at diagnosis was $44.9 \pm 15.8$ years and the female to male ratio was 3.1:1. APS was primary in 82 patients while 78 patients had concomitant rheumatic diseases (SLE in $95 \%$ of patients). $130(81 \%)$ patients had thromboembolic manifestations, $20(13 \%)$ had obstetric morbidities and $10(6 \%)$ had both In patients with secondary APS, $23 \%$ thrombotic or obstetric manifestations occurred before diagnosis of the rheumatic diseases (ie. evolved from primary APS). Lupus anticoagulant was present in $81(51 \%)$ patients, moderate/high titers of anti-cardiolipin were present in $90(56 \%)$ patients and anti- $\beta$ glycoprotein-1 was present in $6(4 \%)$ patients. Sixteen (10\%) patients were double positive while 1 patient was triple positive for these aPL antibodies. Among the thromboembolic manifestations, arterial thrombosis $(n=84)$ (ischemic stroke $77 \%$, myocardial infarction $4.8 \%$, peripheral vascular disease with limb/ digital gangrene $2.4 \%$, retinal artery $2.4 \%$, splenic artery $1.2 \%$, bowel infarct $1.2 \%)$ was more common than venous thrombosis $(n=73)$ (calf vein thrombosis \pm pulmonary embolism $86 \%$, cerebral veins $2.7 \%$, inferior vena cava/ iliac veins $6.8 \%$, retinal vein $2.7 \%$, splenic vein $1.4 \%$ ). The following treatment regimens were used in our APS patients: warfarin (71\%), aspirin plus subcutaneous heparin $(4 \%)$, aspirin + warfarin $(3.1 \%)$, aspirin alone $(17 \%)$ and the direct oral anticoagulant (DOAC) (5\%). Bleeding complications developed in $35(22 \%)$ patients. Over a mean follow-up of $9.9 \pm 7.0$ years, recurrence of thromboembolic or obstetric manifestations recurred in $39(24 \%)$ and $6(4 \%)$ patients respectively. A total of $29(18 \%)$ patients succumbed (median time to death: 6.4 years) and the causes of death were: pneumonia (24\%), septicemia (17\%), intracranial hemorrhage (14\%), myocardial infarction $(10 \%)$, ischemic stroke $(3 \%)$, bowel infarct $(3 \%)$, pulmonary hypertension $(6.9 \%)$ and sudden death with unknown causes (14\%). 